\title{
NAFLD Epidemiology, Emerging Pharmacotherapy, Liver Transplantation Implications and the Trends in the United States
}

\author{
Chiranjeevi Gadiparthi ${ }^{1}$, Moshe Spatz ${ }^{2}$, Simi Greenberg ${ }^{3}$, Umair Iqbal*4 ${ }^{4}$, Sowjanya Kanna ${ }^{5}$, \\ Sanjaya K Satapathy ${ }^{6}$, Arkady Broder ${ }^{1}$ and Aijaz Ahmed ${ }^{7}$
} ${ }^{1}$ Division of Gastroenterology, Hepatology and Clinical Nutrition, Saint Peter's University Hospital, New Brunswick, NJ, USA;
${ }^{2}$ Rutgers Robert Wood Johnson Medical School, Piscataway, NJ, USA; ${ }^{3}$ Nova Southeastern University College of Osteopathic
Medicine, Davie, FL, USA; ${ }^{4}$ Geisinger Commonwealth School of Medicine, Danville, PA, USA; ${ }^{5}$ Division of Gastroenterology,
Allegheny Health Network, Tarentum, PA, USA; ${ }^{6}$ Northwell Health, Division of Hepatology \& Sandra Atlas Bass Center for Liver
Diseases, Manhasset, NY, USA; ${ }^{7}$ Stanford University School of Medicine, Division of Gastroenterology and Hepatology, Stanford, CA, USA

\begin{abstract}
Nonalcoholic fatty liver disease (NAFLD) is a hepatic manifestation of metabolic syndrome. The spread of obesity worldwide in pandemic proportions has led to a rapid rise of NAFLD in developed and developing countries alike. There are no approved pharmacological agents to treat steatohepatitis or advanced fibrosis but obeticholic acid recently has shown some promise in phase III trial. Currently, NAFLD is the number one etiology for simultaneous liver and kidney transplantation in the USA, second most common indication for liver transplantation (LT) and projected to become number one very soon. LT for NAFLD poses unique challenges, as these patients are generally older, obese and more likely to have a number of metabolic risk factors. Bariatric surgery is an option and can be considered if a structured weight loss program does not achieve the sustained weight loss goal. Comprehensive cardiovascular risk assessment and aggressive management of comorbid conditions are crucial in the LT evaluation process to improve post-transplant survival. Recurrent nonalcoholic steatohepatitis after LT is not uncommon, and thus warrants primary and secondary prevention strategies through a multidisciplinary approach. Prevalence of NAFLD in a donor population is a unique and growing concern that limits the access to quality liver grafts.
\end{abstract}

Citation of this article: Gadiparthi C, Spatz M, Greenberg S, Iqbal U, Kanna S, Satapathy SK, et al. NAFLD epidemiology, emerging pharmacotherapy, liver transplantation implications and the trends in the United States. J Clin Transl Hepatol 2020;8(2):215-221. doi: 10.14218/JCTH.2020.00014.

Keywords: NAFLD; NAFL; NASH; Liver transplantation.

Abbreviations: ALD, alcoholic liver disease; CLD, chronic liver disease; CVD, cardiovascular disease; HBV, hepatitis B virus; $\mathrm{HCV}$, hepatitis $\mathrm{C}$ virus; $\mathrm{HCC}$, hepatocellular carcinoma; LT, liver transplantation; NAFLD, nonalcoholic fatty liver disease; NAFL, nonalcoholic fatty liver; NASH, nonalcoholic steatohepatitis; UNOS, United Network for Organ Sharing; OPTN, Organ Procurement and Transportation Network; T2MD, type 2 diabetes mellitus.

Received: 1 March 2020; Revised: 15 April 2020; Accepted: 5 May 2020

*Correspondence to: Umair Iqbal, Geisinger Commonwealth School of Medicine, Danville, PA 17822, USA. Tel: +1-607-282-2759, E-mail: uiqbal@geisinger.edu

\section{Introduction}

Recent advances in chronic hepatitis B and C therapies, combined with increasing prevalence of the obesity epidemic and of other metabolic disorders, such as type 2 diabetes mellitus (T2DM) and hyperlipidemia, have led to a dramatic rise in nonalcoholic fatty liver disease (NAFLD). Today, NAFLD is a major global health problem and has emerged as the $2^{\text {nd }}$ most common indication for liver transplantation (LT) in the USA, and is projected to become number one soon. ${ }^{1}$ NAFLD is a spectrum of liver disease that includes two major types: nonalcoholic fatty liver (NAFL), when there is steatosis, and nonalcoholic steatohepatitis (NASH), when there is significant inflammation. Because of sedentary lifestyle and poor dietary habits, combined with advancing age, prevalence of NAFL and its progression to NASH cirrhosis, liver failure, and HCC, and ultimately the need for LT, are continuing to rise.

While LT is curative and has been shown to improve survival of patients with advanced liver disease of any etiology, there are unique challenges in NASH patients. First, there is no effective pharmacotherapy currently available to halt progression of NASH to advanced fibrosis stages, unlike viral hepatitis. Second, NASH patients are often older, obese and have numerous comorbidities compared to those with other chronic liver disease (CLD) etiologies, thus increasing the risk of mortality during and after LT. Third, increased prevalence of NAFLD in the donor population may adversely affect the availability and quality of liver grafts in future. Finally, recurrent NASH after LT in recipients can negatively affect graft and patient survival. In this evolving landscape, the purpose of this review is to discuss the burden of NAFLD, its risk factors, and its implications on LT.

\section{Epidemiology of NAFLD}

As noted previously, due to the growing obesity epidemic now affecting more than 1.9 billion adults globally, NAFLD has become one of the leading causes of CLD. ${ }^{2}$ According to recent estimates, NAFLD affects as many as one billion individuals throughout the world. Similarly, in the USA, NAFLD affects nearly 80-100 million individuals, making it the number one etiology of CLD. ${ }^{3}$ Nearly $25 \%$ of patients with 
NAFL progress to NASH; however, the true prevalence of biopsy-proven $\mathrm{NASH}$ is difficult to determine, as the majority of NAFL patients do not undergo biopsy. Although the prevalence of NAFLD is increasing throughout the world, there appears to be a significant geographical variation. Overall global prevalence of NAFLD is reported to be $25.2 \%$, according to a recent meta-analysis, with the highest rates being in the Middle East (32\%) and South America (31\%) and the lowest in Africa (14\%). ${ }^{4}$ The prevalence is $27 \%$ in Asia, followed by $24 \%$ in North America, and $23 \%$ in Europe.

The prevalence of $\mathrm{NASH}$ in the general population is estimated to be in the range of $1.5 \%$ and $6.45 \%{ }^{2}$ However, the true prevalence of NASH is difficult to ascertain, primarily because of inaccuracies of diagnostic modalities used. Ultrasound fails to identify the mild form of liver steatosis; up to $50-80 \%$ of patients with NAFLD may have normal liver enzymes, and the gold standard liver biopsy suffers considerable sampling error. ${ }^{5}$ Nevertheless, if the current trend continues, the future burden of $\mathrm{NASH}$ and its related complications are only going to rise astronomically. Novel methodologies used in modeling studies have given a glimpse into future projections. For example, in a modeling study utilizing obesity (by body mass index) and incidence of T2DM in eight countries, involving approximately one-quarter of the world's population, Estes and colleagues ${ }^{6}$ estimated a significant rise in NASH and related complications by 2030 . Specifically, they reported a $63 \%$ increase in NASH prevalence, $168 \%$ increase in incidence of decompensated NASH cirrhosis, $137 \%$ increase in hepatocellular carcinoma incidence, and $178 \%$ increase in liver-related death, with overall number of deaths as high as 800,000 . Figure 1 summarizes the natural history and progression of NAFL/NASH to related CLD, LT and recurrent NAFL/NASH.

\section{Risk factors of NAFLD}

It is very well established that T2DM, obesity and related metabolic syndrome (hyperlipidemia, increased waist circumference, hypertension) play a major role in the pathogenesis of NAFLD. According to a large systematic review, involving 222,816 diabetic patients from 25 countries, the NAFLD prevalence in T2DM patients is as high as $61.1 \% .^{7}$ Similarly, the prevalence of NASH and advanced fibrosis $(\geq \mathrm{F} 3)$ in biopsied diabetic patients was reported as $64 \%$ and $10.4 \%$ respectively. 7,8 NAFLD prevalence increases with increasing body mass index ${ }^{8}$ and it is estimated that $95 \%$ of morbidly

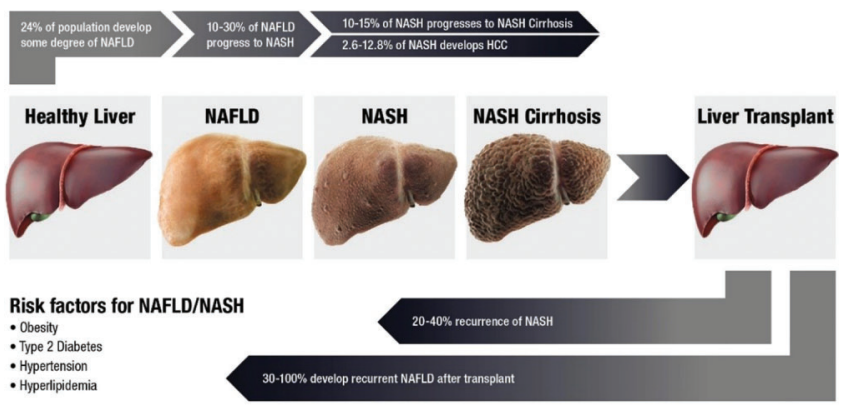

\section{Prevalence and Recurrence of NAFLD/NASH}

Fig. 1. Natural history of nonalcoholic fatty liver disease/nonalcoholic steatohepatitis and recurrence after liver transplantation. obese patients undergoing weight-loss surgery have NAFLD. ${ }^{9}$ As the rates of obesity amongst children have risen from $5.0 \%$ in 1960 to $16.9 \%$ in 2010, NAFLD is increasingly diagnosed in children and adolescents. ${ }^{10}$

Among the non-modifiable risk factors, age, sex and ethnicity are implicated in the pathogenesis of NAFLD. In the USA, NAFLD is most prevalent in Hispanic Americans, followed by non-Hispanic Whites, and is least common in African Americans. ${ }^{11}$ According to population studies, NAFLD is more common in males and prevalence increases with age. NAFLD also causes substantial economic impact due to health care dollar spending. The 10 -year burden of NAFLD is estimated to reach more than 1 trillion dollars in the USA alone. In Europe, the 10-year burden is expected to reach 334 billion euros. ${ }^{10}$

\section{NASH as an indication for LT}

As already noted, NAFLD is now the most common cause of CLD in the USA and Europe and is continuing to rise worldwide. ${ }^{10}$ Among the top indications for LT in the USA, based on the United Network for Organ sharing (UNOS) data from 2003-2014, NASH experienced the highest rate of increase $(162 \%)$ compared to alcohol $(55 \%)$ and hepatitis $\mathrm{C}$ virus (HCV) (33\%). ${ }^{12}$ Subsequently in 2013, NASH became the $2^{\text {nd }}$ leading indication for LT in the USA. ${ }^{1}$ In the same year, the advent of direct acting antiviral agents, a highly effective and safer type of medications, has led to dramatic reduction in chronic HCV disease burden and rates of LT. The decline of $\mathrm{HCV}$ prevalence, combined with recent resurgence of alcoholism, has resulted in alcoholic liver disease (ALD) to become the number one cause for LT in the USA, surpassing HCV. ${ }^{13}$ However, this trend is not expected to last very long, as $\mathrm{NASH}$, with its current trajectory, is expected to replace ALD and become the leading indication very soon.

Although NASH patients undergoing LT are older and obese compared to those with other etiologies, studies have demonstrated that the short-term and long-term post-transplant survival rates are very similar. For example, 1- and 3-year post-transplant survival rates for NASH LT recipients were $84 \%$ and $78 \%$ compared to $87 \%$ and $78 \%(p=0.67)$ for other indications (HCV, ALD, and cholestatic and autoimmune hepatitis). In addition, the 3-year graft survival rate was $76 \%$ for NASH LT recipients. ${ }^{14}$ In another study, the 5-year survival of LT recipients for NASH was superior to those with HCV (77.81\% vs. $72.15 \%) .{ }^{12}$ More recently, in a retrospective study of 26,121 LT recipients with HCC from 2002-2016, NASH patients were older (mean age of 62.9 years) compared to those with $\mathrm{HCV}$ (59.2 years), HBV (57.2 years) and ALD (60.6 years), obese (body mass index of $>30, \mathrm{NASH}$ $60.5 \%$ vs. HCV $32.9 \%$, HBV $14.4 \%$ and ALD $40 \%$ ) and more likely to be diabetic (NASH $60.3 \%$ vs. HCV $22.5 \%$, HBV $19.3 \%$ and ALD $32.7 \%$ ). The 1 -year post-transplant survival rate was similar across all cohorts $(p>0.5)$ but long-term mortality and graft loss were highest in HCV and lowest in HBV. ${ }^{15}$

Although HCV has remained as the most common cause of HCC in LT candidates, NASH was the most rapidly growing cause, with 11.8 -fold increase from 2002 to 2016 . Figures 2 and 3 demonstrate the temporal trends of annual waitlist additions and LT rates in the USA from 2008-2018 for the top 5 etiologies of CLD based on the most recent UNOS data. These graphs demonstrate a steady and upward trend for NASH-related LT in the USA. 


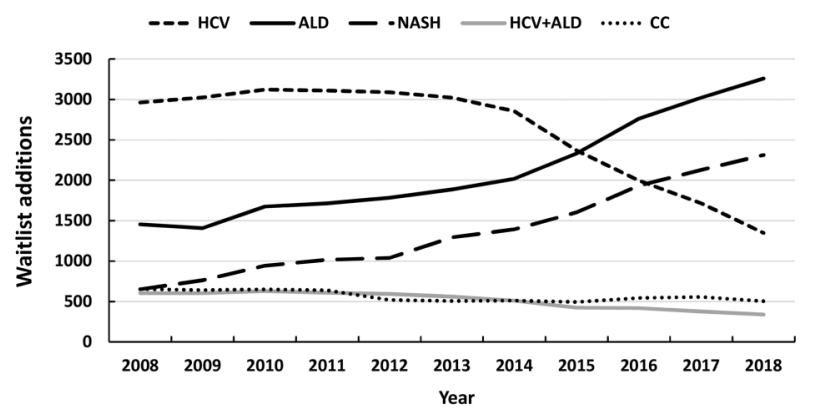

Fig. 2. Temporal trends in annual waitlist additions for top 5 etiologies in the USA UNOS 2008-2018.

Abbreviations: ALD, alcoholic liver disease; CC, cryptogenic cirrhosis; $\mathrm{HCV}$, hepatitis C virus; NASH, nonalcoholic steatohepatitis; UNOS, United Network for Organ Sharing.

\section{Strategies to prevent progression of NAFL/NASH}

T2DM, insulin resistance, obesity and other metabolic risk factors are the main factors driving the prevalence of NAFLD and remain the primary targets in its prevention and progression. Lifestyle modifications, such as exercise and a healthy diet, resulting in sustained weight loss are the only proven and effective strategies available currently to curtail the NAFL/NASH burden. ${ }^{2}$ NAFLD is considered to be the hepatic manifestation metabolic syndrome. Approximately $70 \%$ of patients with T2DM have NAFLD and these diseases share common pathophysiological pathways.

Antidiabetic drugs, as well as statins, can improve biochemical and histological features of NAFL/NASH. ${ }^{16}$ Management of comorbidities is not only critical in decreasing progression of NASH but is also pivotal in decreasing cardiovascular mortality, which is the major cause of death in these patients. While weight loss is the single most effective intervention, it can be extremely challenging for patients to achieve the sustained weight loss goal. Structured weight management programs with a multidisciplinary team have had variable success. In morbidly obese patients, who are less likely to be considered for LT surgery, bariatric surgery may be necessary and has not shown to have any negative impact on the LT outcomes. ${ }^{17}$ Additionally, bariatric surgery improves and sometimes eliminates other comorbid conditions in many patients and has shown to improve long-term survival from the two most common causes of death in NAFLD, malignancy and cardiovascular disease (CVD). ${ }^{18}$

\section{Recent advances in pharmacotherapy}

The two primary endpoints of numerous ongoing clinical trials are resolution of steatohepatitis and improvement in liver fibrosis or both, which are considered surrogate markers for slowing the progression of NASH. Despite the vast knowledge of risk factors and clear elucidation of pathophysiologic pathways in $\mathrm{NASH}$, there has been no significant breakthrough in disease-specific pharmacotherapy yet. However, recent studies have shown some promise. Obeticholic acid, which is already approved for primary biliary cholangitis, is currently under review by the national Federal Drug Administration and could potentially be the first approved medication for NASH. In recent interim analysis of the REGENERATE trial, a phase III multicenter, randomized placebo-controlled study, biopsy- proven NASH patients with F1-F3 fibrosis, treatment with obeticholic acid at $25 \mathrm{mg}$ a day has reached the primary end point of improvement in liver fibrosis score by $\geq 1$ stage without worsening of NASH at 18 months follow-up compared to placebo ( $23 \%$ vs. $12 \%, p=0.0002) .{ }^{19}$

A number of other agents, such as cenicriviroc, elafibranor, aramchol and resmetirom, are in the pipeline with phase III trials. Primary end points are fibrosis improvement and prevention of worsening of NASH for cenicriviroc and NASH resolution and prevention of progression fibrosis for elafibrinor. ${ }^{20}$ Elafibranor with its favorable safety profile and tolerability makes an attractive choice, but the phase III results of the RESOLVE-IT trial are delayed. Cenicriviroc's phase IIb results were promising, but poor preliminary efficacy results cast doubt over the success of the ongoing AURORA phase III trial. ${ }^{21}$ The strong safety and efficacy found in a phase II trial as well as in preliminary results of the phase III MAESTRO$\mathrm{NASH}$ trial has made resmetirom a hopeful alternative. ${ }^{22}$ Finally, early results of aramchol are inconsistent but phase III trial is in progress.

\section{Special considerations of NASH in LT}

$\mathrm{NASH}$ is a multisystem disease associated, with clinical manifestations beyond the liver. NASH patients are at higher risk of mortality and morbidity due to increased prevalence of metabolic comorbidities. Importantly, CVD and malignancies contribute to higher mortality in NASH patients than liverrelated morality (cirrhosis and $\mathrm{HCC}$ ). ${ }^{23}$ These factors should be considered in the LT evaluation process.

\section{Cardiovascular risk assessment and management in LT}

NASH independently contributes to increased cardiovascular mortality and morbidity, regardless of other cardiovascular risk factors. In addition to coronary artery disease, several other cardiovascular complications are reported in NAFLD patients, such as premature atherosclerosis to left ventricular dysfunction and hypertrophy, aortic sclerosis, congestive heart failure, and cardiac arrythmias (atrial fibrillation and prolonged QTc). ${ }^{24}$ Based on the recent meta-analysis by Targher et al., ${ }^{25}$ including 34000 patients, presence of NAFLD is associated with $65 \%$ increase in fatal and nonfatal cardiovascular events at medial 7-year follow-up period. LT surgery is inherently stressful to the heart because of sudden changes in hemodynamic parameters and furthermore, post-operative complications can unmask underlying clinically silent CVD leading to poor outcomes and increased mortality. ${ }^{26}$ Compared to other etiologies, post-transplant cardiovascular events are higher in LT recipients with NASH cirrhosis, especially in the immediate postoperative period. ${ }^{27}$ Therefore, several societies recommend comprehensive cardiovascular risk assessment and testing during the LT evaluation process.

While it is important to thoroughly evaluate these patients, it is unclear what constitutes comprehensive cardiovascular evaluation and that itself varies significantly across the LT centers. However, the general approach should focus on identifying underlying CVD, congestive heart failure and portopulmonary hypertension, and optimize these conditions prior to LT surgery and exclude high-risk patients. Patients with clinically significant congestive heart failure should be excluded from LT due to the risk of decompensation and death. High-risk patients with coronary artery disease should 


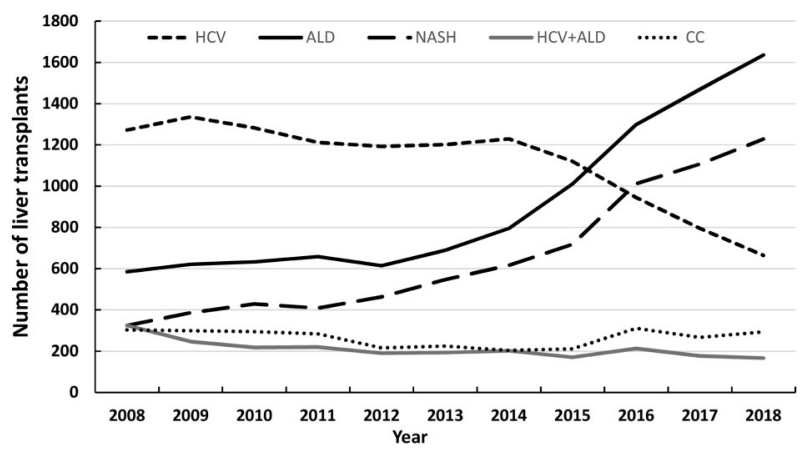

Fig. 3. Temporal trends of annual liver transplantations for top 5 etiologies in the USA UNOS 2008-2018.

Abbreviations: ALD, alcoholic liver disease; CC, cryptogenic cirrhosis; $\mathrm{HCV}$, hepatitis C virus; NASH, nonalcoholic steatohepatitis; UNOS, United Network for Organ Sharing.

either undergo revascularization before transplant surgery or be excluded from the waitlist. ${ }^{28}$ Additionally, patients with moderate to severe portopulmonary hypertension who fail to respond to vasodilator therapy are considered high-risk and should be excluded from LT. ${ }^{29}$

Both structural and functional cardiac evaluation is required in LT candidates. Cardiopulmonary exercise testing is challenging because cirrhotic patients may be deconditioned with poor performance status, ascites, malnutrition, and frailty. Doppler echocardiography is routinely performed to assess left and right ventricular and valvular function and to screen for pulmonary hypertension. Noninvasive stress testing is performed using dobutamine stress echocardiogram or myocardial perfusion imaging, and if abnormal, further evaluation with cardiac catheterization may be required. Although, dobutamine stress echocardiogram has shown to be a good noninvasive test to evaluate coronary ischemia in the general population, it suffers from poor sensitivity in cirrhotic patients due to difficulty in achieving target heart rate and double product, perhaps due to use of betablockers for variceal prophylaxis in many patients. ${ }^{30}$ Because of these limitations, several centers routinely perform right and left heart catheterization as a part of transplant evaluation process of NASH patients. Although this approach is debated, it may be reasonable in these patients due to their inherently higher cardiovascular risk. Furthermore, this approach allows identifying patients with clinically silent coronary artery disease and provides an opportunity to revascularize them prior to LT. However, it is interesting to note that in a small study of 13 patients, 50\% with severe coronary artery disease died due to cardiovascular causes after LT surgery despite undergoing revascularization ( 3 percutaneous coronary intervention and 6 coronary artery bypass graft surgery) prior to surgery. ${ }^{31}$ This suggests that there may be other factors to consider before embarking on LT surgery in NASH patients.

Other traditional risk factors, such as hypertension, hyperlipidemia, T2DM and CKD should be screened and managed appropriately. Despite the small potential risk of hepatotoxicity, statins have far reaching benefits against progression of cirrhosis, portal hypertension and HCC beyond treating hyperlipidemia and thus should be strongly considered. ${ }^{32}$ Finally, the LT evaluation process should include a multidisciplinary team approach, including cardiology, cardiac anes- thesiology, nephrology, endocrinology and nutrition in addition to hepatology and transplant surgery, to appropriately risk stratify and optimize NASH patients to improve post-transplant outcomes.

\section{Obesity and role of bariatric surgery}

It is clear that obesity is highly prevalent in NASH patients. Obesity by itself and high body mass index are not absolute contraindications for LT surgery. Moreover, body mass index is not a reliable indicator and tends to overestimate obesity in the presence of ascites and volume overload. Studies on effects of obesity on LT outcomes have shown conflicting results. In a large meta-analysis published in 2015 that included 13 studies comparing 2275 obese patients with 72212 non-obese LT recipients, body mass index did not negatively impact the post-transplant survival. ${ }^{33}$ Because body mass index is a less reliable surrogate for obesity, other parameters such as visceral adipose tissue and muscle mass should be included in the evaluation process to optimally predict post-transplant survival. ${ }^{34}$

Based on the Scientific Registry of Organ Transplants data from 1994 to 2013 that included over 85,000 adult LT recipients, body mass index values did not impact posttransplant survival, whereas T2DM in LT recipients in both pre-transplant (hazard ratio of $1.21,95 \%$ confidence interval of $1.12-1.30$ ) and post-transplant settings (hazard ratio of $1.06,95 \%$ confidence interval of $1.02-1.11$ ) and T2DM in donors (hazard ratio of $1.10 ; 95 \%$ confidence interval of 1.02-1.19) were associated with poor outcomes. ${ }^{35}$ Therefore, it is evident that, not obesity alone, but the presence of other metabolic comorbidities in addition to obesity lead to poorer outcomes. Nevertheless, a body mass index of $\geq 40$ is generally considered a relative contraindication for $L T$ in most centers.

Bariatric surgery is a feasible option for morbidly obese patients. Due to lack of data regarding long-term follow-up, the optimal timing of bariatric surgery whether before, during or after LT surgery remains unclear. Although metabolic comorbidities seem to improve with bariatric surgery in the pre-transplant setting, studies have reported significantly higher post-operative complications. ${ }^{36,37}$ Concomitant LT and bariatric surgery is an option in highly select patients. In a Mayo study, LT candidates with a body mass index of $\geq 35$ who were unable to achieve weight loss goal in the pre-transplant setting underwent combined LT surgery and sleeve gastrectomy. ${ }^{38}$ Compared to the LT alone group, the combined LT-sleeve gastrectomy group achieved more sustained weight loss at 3 -year post-transplant follow-up and were less likely to develop insulin resistance, hepatic steatosis and hypertension. However, this approach is limited by prolonged surgery time, immediate need for immunosuppression post-transplant, and risk of poor nutrition status. ${ }^{39}$ Overall, based on studies, sleeve gastrectomy appears to be a preferred option compared to Roux-en-Y gastric bypass surgery and has several advantages. Compared to Roux-en$Y$ gastric bypass, sleeve gastrectomy requires less operative time, does not cause malabsorption or altered anatomy, maintains adequate immunosuppression, and provides endoscopic access to the biliary system in the event of post-operative biliary complications. ${ }^{26}$ While weight-loss is a desired goal in NASH patients, it is important to screen and manage malnutrition and sarcopenia, which are independent predictors of poor waitlist and post-transplant mortality. Studies 
have shown that up to $25 \%$ of obese patients suffer from malnutrition. ${ }^{40}$ Therefore, it is very critical to maintain proper nutrition and adequate protein supplementation in obese NASH patients who undergo bariatric surgery or are enrolled in weight-loss programs, to avoid malnutrition and sarcopenia.

\section{Post-transplant outcomes of NASH compared other CLDs}

Despite the higher prevalence of cardiovascular risk factors in NASH patients, several studies have shown that post-transplant outcomes of NASH patients are similar to those of other indications of LT. In a large systematic review comparing LT recipients with NASH $(n=717)$ and non-NASH $(n=3520), 1-$ year, 3-year, and 5-year post-transplant survival rates were similar between the two groups, although the NASH patients were older, with higher prevalence of women, had higher body mass index, and were more likely to have T2DM, hypertension and hyperlipidemia compared to non-NASH counterparts. ${ }^{41}$ While NASH patients experienced higher mortality due to CVD (odds ratio of $1.65,95 \%$ confidence interval of $1.01-2.70 ; p=0.05$ ) and sepsis (odds ratio of $1.71 ; 95 \%$ confidence interval of $1.17-2.50 ; p=0.006)$, graft failure was lower (odds ratio of $0.21 ; 95 \%$ confidence interval of $0.05-0.89 ; p=0.03$ ) compared to non-NASH LT recipients. Studies based on UNOS data, showed similar results. From 2001 to 2009, comparing 1959 NASH LT recipients and 33,822 non-NASH patients, 1 -year and 3-year post-LT survival was similar: $84 \%$ and $78 \%$ for NASH respectively, $86 \%$ and $79 \%$ for cryptogenic cirrhosis, and $87 \%$ and $78 \%$ for other indications $(p=0.67) .{ }^{14}$ A more recent UNOS study from 2003 to 2014 showed outcomes of 63,061 adult LT recipients, including 20,782 HCV patients (32.96\%), 9470 ALD patients $(15.02 \%)$, and $8262 \mathrm{NASH}$ patients $(13.11 \%)$. Results of this study demonstrated that 5-year post-transplant survival was better in NASH patients compared to HCV (77.81\% vs. $72.15, p<0.001)$ despite the NASH cohort being more likely to have obesity and higher rates of T2DM and CVD. ${ }^{12}$

In another retrospective study, Sadler et al. ${ }^{42}$ showed that HCC patients with NASH $(60 / 929,6.5 \%)$ and non-NASH $(869 / 929,93.5 \%)$ had similar 1-year, 3-year, 5-year survival rates $(98 \%, 96 \%$, and $80 \%$ respectively in NASH vs. $95 \%$, $84 \%$, and $78 \%$ in non-NASH, $p=0.1$ ). Overall, based on multiple studies, both single-center as well as those involving large databases, NASH patients, despite the older age and higher prevalence of comorbidities compared to other etiologies, showed similar post-transplant survival. This could, inpart, be explained by better graft survival rates and rigorous LT selection process, where patients with higher cardiovascular risk are excluded from the LT waitlist. ${ }^{26}$

\section{Recurrent NASH after LT}

While recurrent NASH is an important complication in LT recipients with $\mathrm{NASH}$, de novo $\mathrm{NASH}$ is a growing concern in non-NASH LT recipients. There are several reasons for this: patients in the post-transplant setting generally feel well, not in a catabolic state of cirrhosis, and are more likely increase the daily calorie intake, resulting in accelerated weight gain. In fact, use of corticosteroids and other immunosuppressive agents, including calcineurin inhibitors and mammalian target rapamycin inhibitors can result in metabolic derangement and development of obesity, insulin resistance, T2DM, hypertension, and hyperlipidemia.43-46 Additionally, some studies have suggested that non-NASH indications, such as HCV and ALD, are also associated with development of de novo $\mathrm{NASH}$ after $\mathrm{LT}^{47,48}$ There exists a considerable heterogeneity among the studies that estimated the prevalence of recurrent and de novo NASH. In a study, $30 \%$ of the LT recipients with $\mathrm{NASH}$ developed recurrent steatohepatitis at 1-year; however, none of them developed cirrhosis in long-term follow-up. ${ }^{49}$ The rates of recurrent NAFL in another study, comprising $257 \mathrm{NASH} /$ cryptogenic cirrhosis LT recipients, at 1-year, 2-year, 5-year, and 10-year follow-up were higher $(8.2 \%, 13.6 \%, 24.9 \%$, and $32.9 \%$ respectively) compared to non-NASH/cryptogenic cirrhosis LT recipients $(3.1 \%$, $5.9 \%, 9.6 \%$, and $10 \%) .{ }^{50}$ However, the rate of recurrent NASH was much lower, at $5 \%$ (13 out of 257), and advanced fibrosis was rare, and in fact, post-LT survival was similar to that in the non-NASH/cryptogenic cirrhosis group. Nevertheless, CVD and infection-related complications were higher in patients with recurrent NAFL. Therefore, these patients should be closely monitored to prevent rapid weight gain, and screened for development of metabolic conditions and managed accordingly.

Presence of NAFLD in donor livers is another challenge, due to overall increasing prevalence of NAFLD in the general population. This ominous trend not only affects the quality and numbers of donor livers but it also may cause delayed or primary graft dysfunction, as well as graft loss and poor recipient outcomes ultimately. ${ }^{51-54}$ Steatosis-induced microcirculatory and cellular dysfunction following reperfusion is thought to be a major cause for hepatocyte necrosis and graft loss. Thus, it is important to identify the extent of steatosis in donor livers. Mild steatosis $(<30 \%)$ in the donor grafts is generally accepted and not associated with poor outcomes compared to more than $>30 \%$, which showed poor outcomes at 1 -year after transplant. ${ }^{51,52,54}$ Some transplant centers routinely perform donor rush liver biopsy prior to LT and discard high-risk grafts; however, this approach may prolong cold-ischemia time and is not widely available in all centers. Nonetheless, donor steatosis does not appear to affect rates of recurrent NASH in NASHrecipients more than non-NASH recipients and therefore, there is insufficient data to recommend different approach in each group at this time.

\section{Conclusions}

NAFLD is steadily raising throughout the world and is on a trajectory to become the number 1 indication of LT in the USA. Despite the prevalence of metabolic comorbidities, posttransplant survival for NASH is comparable to other etiologies. Obesity alone is not a contraindication for LT in the absence of other comorbid conditions and body mass index is not a reliable indicator of obesity in the presence of ascites and volume overload. Weight loss surgery before or during LT surgery can be considered in select patients but it is limited only to specialized centers, due to higher complication rates. Prevention of sarcopenia and malnutrition while achieving weight loss is a challenging task. LT cures end-stage liver disease but not the underlying metabolic risk factors associated with NAFLD; therefore, strategies to address these comorbidities are crucial to improve outcomes and prevent recurrence of NAFLD after transplantation. Prevalence of 
NAFLD in donor livers is increasing and needs attention to expand the donor pool to meet the growing demand for LT.

\section{Funding}

None to declare.

\section{Conflict of interest}

The authors have no conflict of interests related to this publication.

\section{Author contributions}

Study concept and design, acquisition and review of literature, and drafting and approval of the final manuscript (CG, MS and SG), drafting, supervision, critical revision and approval of the final manuscript (UI, SK, SS, AB and AA). All authors were involved in the final approval of the version of the manuscript submitted and have agreed to be accountable for all aspects of the work.

\section{References}

[1] Wong RJ, Aguilar M, Cheung R, Perumpail RB, Harrison SA, Younossi ZM, et al. Nonalcoholic steatohepatitis is the second leading etiology of liver disease among adults awaiting liver transplantation in the United States. Gastroenterology 2015;148:547-555. doi: 10.1053/j.gastro.2014.11.039.

[2] Younossi ZM, Marchesini G, Pinto-Cortez H, Petta S. Epidemiology of nonalcoholic fatty liver disease and nonalcoholic steatohepatitis: Implications for liver transplantation. Transplantation 2019;103:22-27. doi: 10. 1097/TP.0000000000002484.

[3] Perumpail BJ, Khan MA, Yoo ER, Cholankeril G, Kim D, Ahmed A. Clinical epidemiology and disease burden of nonalcoholic fatty liver disease. World ] Gastroenterol 2017;23:8263-8276. doi: 10.3748/wjg.v23.i47.8263.

[4] Younossi ZM, Koenig AB, Abdelatif D, Fazel Y, Henry L, Wymer M. Global epidemiology of nonalcoholic fatty liver disease-Meta-analytic assessment of prevalence, incidence, and outcomes. Hepatology 2016;64:73-84. doi: 10.1002/hep. 28431

[5] Mahady SE, George J. Predicting the future burden of NAFLD and NASH. J Hepatol 2018;69:774-775. doi: 10.1016/j.jhep.2018.06.025.

[6] Estes C, Anstee QM, Arias-Loste MT, Bantel H, Bellentani S, Caballeria J, et al. Modeling NAFLD disease burden in China, France, Germany, Italy, Japan, Spain, United Kingdom, and United States for the period 2016-2030. J Hepatol 2018;69:896-904. doi: 10.1016/j.jhep.2018.05.036.

[7] Younossi ZM, Golabi P, de Avila L, Paik JM, Srishord M, Fukui N, et al. The global epidemiology of NAFLD and NASH in patients with type 2 diabetes: A systematic review and meta-analysis. J Hepatol 2019;71:793-801. doi: 10. 1016/j.jhep.2019.06.021.

[8] Ong JP, Elariny $H$, Collantes $R$, Younoszai $A$, Chandhoke $V$, Reines $H D$, et al. Predictors of nonalcoholic steatohepatitis and advanced fibrosis in morbidly obese patients. Obes Surg 2005;15:310-315. doi: 10 . 1381/0960892053576820.

[9] Lonardo A, Ballestri S, Marchesini G, Angulo P, Loria P. Nonalcoholic fatty liver disease: a precursor of the metabolic syndrome. Dig Liver Dis 2015;47:181190. doi: 10.1016/j.dld.2014.09.020.

[10] Younossi Z, Anstee QM, Marietti M, Hardy T, Henry L, Eslam M, et al. Globa burden of NAFLD and NASH: trends, predictions, risk factors and prevention. Nat Rev Gastroenterol Hepatol 2018;15:11-20. doi: 10.1038/nrgastro. 2017.109.

[11] Williams CD, Stengel J, Asike MI, Torres DM, Shaw J, Contreras M, et al. Prevalence of nonalcoholic fatty liver disease and nonalcoholic steatohepatitis among a largely middle-aged population utilizing ultrasound and liver biopsy: a prospective study. Gastroenterology 2011;140:124-131. doi: 10 . 1053/j.gastro.2010.09.038.

[12] Cholankeril G, Wong RJ, Hu M, Perumpail RB, Yoo ER, Puri P, et al. Liver transplantation for nonalcoholic steatohepatitis in the US: Temporal trends and outcomes. Dig Dis Sci 2017;62:2915-2922. doi: 10.1007/s10620-0174684-x.

[13] Cholankeril G, Ahmed A. Alcoholic liver disease replaces hepatitis C virus infection as the leading indication for liver transplantation in the United States. Clin Gastroenterol Hepatol 2018;16:1356-1358. doi: 10.1016/j. cgh.2017.11.045.
[14] Charlton MR, Burns JM, Pedersen RA, Watt KD, Heimbach JK, Dierkhising RA. Frequency and outcomes of liver transplantation for nonalcoholic steatohepatitis in the United States. Gastroenterology 2011;141:1249-1253. doi: 10.1053/j.gastro.2011.06.061.

[15] Younossi Z, Stepanova M, Ong JP, Jacobson IM, Bugianesi E, Duseja A, et al. Nonalcoholic steatohepatitis is the fastest growing cause of hepatocellula carcinoma in liver transplant candidates. Clin Gastroenterol Hepatol 2019; 17:748-755.e3. doi: 10.1016/j.cgh.2018.05.057.

[16] Katsiki N, Athyros VG, Mikhailidis DP. Non-alcoholic fatty liver disease in patients with type 2 diabetes mellitus: Effects of statins and antidiabetic drugs. J Diabetes Complications 2017;31:521-522. doi: 10.1016/j.jdiacomp.2016.12.006

[17] Safwan M, Collins KM, Abouljoud MS, Salgia R. Outcome of liver transplantation in patients with prior bariatric surgery. Liver Transpl 2017;23:14151421. doi: $10.1002 /$ It.24832

[18] Chalasani N, Younossi Z, Lavine JE, Charlton M, Cusi K, Rinella M, et al. The diagnosis and management of nonalcoholic fatty liver disease: Practice guidance from the American Association for the Study of Liver Diseases. Hepatology 2018;67:328-357. doi: 10.1002/hep.29367.

[19] Younossi ZM, Ratziu V, Loomba R, Rinella M, Anstee QM, Goodman Z, et al. Obeticholic acid for the treatment of non-alcoholic steatohepatitis: interim analysis from a multicentre, randomised, placebo-controlled phase 3 trial. Lancet 2019;394:2184-2196. doi: 10.1016/S0140-6736(19)33041-7.

[20] Alukal JJ, Thuluvath PJ. Reversal of NASH fibrosis with pharmacotherapy. Hepatol Int 2019;13:534-545. doi: 10.1007/s12072-019-09970-3.

[21] Friedman SL, Ratziu V, Harrison SA, Abdelmalek MF, Aithal GP, Caballeria J, et al. A randomized, placebo-controlled trial of cenicriviroc for treatment of nonalcoholic steatohepatitis with fibrosis. Hepatology 2018;67:1754-1767. doi: 10.1002/hep.29477.

[22] Harrison SA, Bashir MR, Guy CD, Zhou R, Moylan CA, Frias JP, et al. Resmetirom (MGL-3196) for the treatment of non-alcoholic steatohepatitis: a multicentre, randomised, double-blind, placebo-controlled, phase 2 trial. Lancet 2019;394:2012-2024. doi: 10.1016/S0140-6736(19)32517-6.

[23] Byrne CD, Targher G. NAFLD: a multisystem disease. ] Hepatol 2015;62: S47-S64. doi: 10.1016/j.jhep.2014.12.012.

[24] Lonardo A, Nascimbeni F, Mantovani A, Targher G. Hypertension, diabetes, atherosclerosis and NASH: Cause or consequence? J Hepatol 2018;68:335352. doi: 10.1016/j.jhep.2017.09.021.

[25] Targher G, Byrne CD, Lonardo A, Zoppini G, Barbui C. Non-alcoholic fatty liver disease and risk of incident cardiovascular disease: A meta-analysis. J Hepatol 2016;65:589-600. doi: 10.1016/j.jhep.2016.05.013.

[26] Tsochatzis E, Coilly A, Nadalin S, Levistky ], Tokat Y, Ghobrial M, et al. International liver transplantation consensus statement on end-stage liver disease due to nonalcoholic steatohepatitis and liver transplantation. Transplantation 2019;103:45-56. doi: 10.1097/TP.0000000000002433.

[27] Vanwagner LB, Bhave M, Te HS, Feinglass J, Alvarez L, Rinella ME. Patients transplanted for nonalcoholic steatohepatitis are at increased risk for postoperative cardiovascular events. Hepatology 2012;56:1741-1750. doi: 10. 1002/hep. 25855

[28] Plotkin JS, Johnson LB, Rustgi V, Kuo PC. Coronary artery disease and liver transplantation: the state of the art. Liver Transpl 2000;6:S53-S56. doi: 10. 1002/It.500060511.

[29] Krowka MJ, Fallon MB, Kawut SM, Fuhrmann V, Heimbach JK, Ramsay MA, et al. International liver transplant society practice guidelines: Diagnosis and management of hepatopulmonary syndrome and portopulmonary hypertension. Transplantation 2016;100:1440-1452. doi: 10.1097/TP. 0000000000001229.

[30] Ripoll C, Yotti R, Bermejo J, Bañares R. The heart in liver transplantation. J Hepatol 2011;54:810-822. doi: 10.1016/j.jhep.2010.11.003.

[31] Snipelisky DF, McRee C, Seeger K, Levy M, Shapiro BP. Coronary interventions before liver transplantation might not avert postoperative cardiovascular events. Tex Heart Inst J 2015;42:438-442. doi: 10.14503/THIJ-14-4738.

[32] Vargas JI, Arrese M, Shah VH, Arab JP. Use of statins in patients with chronic liver disease and cirrhosis: Current views and prospects. Curr Gastroenterol Rep 2017;19:43. doi: 10.1007/s11894-017-0584-7.

[33] Saab S, Lalezari D, Pruthi P, Alper T, Tong MJ. The impact of obesity on patient survival in liver transplant recipients: a meta-analysis. Liver Int 2015;35: 164-170. doi: 10.1111/liv.12431.

[34] Barone M, Viggiani MT, Avolio AW, Iannone A, Rendina M, Di Leo A. Obesity as predictor of postoperative outcomes in liver transplant candidates: Review of the literature and future perspectives. Dig Liver Dis 2017;49:957-966. doi: 10.1016/j.dld.2017.07.004

[35] Younossi ZM, Stepanova M, Saab S, Kalwaney S, Clement S, Henry L, et al. The impact of type 2 diabetes and obesity on the long-term outcomes of more than 85000 liver transplant recipients in the US. Aliment Pharmacol Ther 2014;40:686-694. doi: 10.1111/apt.12881.

[36] Takata MC, Campos GM, Ciovica R, Rabl C, Rogers SJ, Cello JP, et al. Laparoscopic bariatric surgery improves candidacy in morbidly obese patients awaiting transplantation. Surg Obes Relat Dis 2008;4:159-164. doi: 10 . 1016/j.soard.2007.12.009. 


\section{Gadiparthi C. et al: Liver transplantation in NASH patients}

[37] Lin MY, Tavakol MM, Sarin A, Amirkiai SM, Rogers SJ, Carter JT, et al. Laparoscopic sleeve gastrectomy is safe and efficacious for pretransplant candidates. Surg Obes Relat Dis 2013;9:653-658. doi: 10.1016/j.soard.2013.02.013.

[38] Zamora-Valdes D, Watt KD, Kellogg TA, Poterucha J], Di Cecco SR, FranciscoZiller NM, et al. Long-term outcomes of patients undergoing simultaneous liver transplantation and sleeve gastrectomy. Hepatology 2018;68:485495. doi: 10.1002/hep.29848.

[39] Heimbach JK, Watt KD, Poterucha J], Ziller NF, Cecco SD, Charlton MR, et al. Combined liver transplantation and gastric sleeve resection for patients with medically complicated obesity and end-stage liver disease. Am J Transplant 2013;13:363-368. doi: 10.1111/j.1600-6143.2012.04318.x.

[40] Leibovitz E, Giryes S, Makhline R, Zikri Ditch M, Berlovitz Y, Boaz M. Malnutrition risk in newly hospitalized overweight and obese individuals: Mr NOI Eur J Clin Nutr 2013;67:620-624. doi: 10.1038/ejcn.2013.45.

[41] Wang X, Li J, Riaz DR, Shi G, Liu C, Dai Y. Outcomes of liver transplantation for nonalcoholic steatohepatitis: a systematic review and meta-analysis. Clin Gastroenterol Hepatol 2014;12:394-402.e1. doi: 10.1016/j.cgh.2013.09. 023.

[42] Sadler EM, Mehta N, Bhat M, Ghanekar A, Greig PD, Grant DR, et al. Liver transplantation for NASH-related hepatocellular carcinoma versus nonNASH etiologies of hepatocellular carcinoma. Transplantation 2018;102: 640-647. doi: 10.1097/TP.0000000000002043.

[43] Davidson J, Wilkinson A, Dantal J, Dotta F, Haller H, Hernández D, et al. Newonset diabetes after transplantation: 2003 International consensus quidelines. Proceedings of an international expert panel meeting. Barcelona, Spain, 19 February 2003. Transplantation 2003;75:SS3-SS24. doi: 10. 1097/01.TP.0000069952.49242.3E.

[44] Pham PT, Pham PM, Pham SV, Pham PA, Pham PC. New onset diabetes after transplantation (NODAT): an overview. Diabetes Metab Syndr Obes 2011;4: 175-186. doi: 10.2147/DMSO.S19027.

[45] Charco R, Cantarell C, Vargas V, Capdevila L, Lázaro JL, Hidalgo E, et al. Serum cholesterol changes in long-term survivors of liver transplantation: a comparison between cyclosporine and tacrolimus therapy. Liver Transpl Surg 1999;5:204-208. doi: 10.1002/It.500050303.

[46] Watt KD, Pedersen RA, Kremers WK, Heimbach JK, Charlton MR. Evolution of causes and risk factors for mortality post-liver transplant: results of the NIDDK long-term follow-up study. Am J Transplant 2010;10:1420-1427. doi: $10.1111 / \mathrm{j} .1600-6143.2010 .03126 . x$.

[47] Dumortier J, Giostra E, Belbouab S, Morard I, Guillaud O, Spahr L, et al. Nonalcoholic fatty liver disease in liver transplant recipients: another story of "seed and soil". Am J Gastroenterol 2010;105:613-620. doi: 10.1038/ajg. 2009.717

[48] Galvin Z, Rajakumar R, Chen E, Adeyi O, Selzner M, Grant D, et al. Predictors of de novo nonalcoholic fatty liver disease after liver transplantation and associated fibrosis. Liver Transpl 2019;25:56-67. doi: 10.1002/It.25338.

[49] Bhagat V, Mindikoglu AL, Nudo CG, Schiff ER, Tzakis A, Regev A. Outcomes of liver transplantation in patients with cirrhosis due to nonalcoholic steatohepatitis versus patients with cirrhosis due to alcoholic liver disease. Liver Transpl 2009;15:1814-1820. doi: 10.1002/lt.21927.

[50] Yalamanchili K, Saadeh S, Klintmalm GB, Jennings LW, Davis GL. Nonalcoholic fatty liver disease after liver transplantation for cryptogenic cirrhosis or nonalcoholic fatty liver disease. Liver Transpl 2010;16:431-439. doi: 10. 1002/It.22004

[51] McCormack L, Dutkowski P, El-Badry AM, Clavien PA. Liver transplantation using fatty livers: always feasible? J Hepatol 2011;54:1055-1062. doi: 10. 1016/j.jhep.2010.11.004.

[52] Angulo P. Nonalcoholic fatty liver disease and liver transplantation. Liver Transpl 2006;12:523-534. doi: 10.1002/lt.20738.

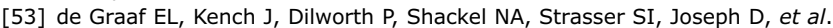
Grade of deceased donor liver macrovesicular steatosis impacts graft and recipient outcomes more than the Donor Risk Index. J Gastroenterol Hepatol 2012;27:540-546. doi: 10.1111/j.1440-1746.2011.06844.x.

[54] Spitzer AL, Lao OB, Dick AA, Bakthavatsalam R, Halldorson JB, Yeh MM, et al. The biopsied donor liver: incorporating macrosteatosis into high-risk donor assessment. Liver Transpl 2010;16:874-884. doi: 10.1002/It.22085. 